\title{
Zygostates alleniana (Orchidaceae: Epidendroideae: Cymbidieae: Oncidiinae): estructura floral relacionada con la polinización
}

\author{
Natalia E. Gómiz ${ }^{1, *}$, Juan P. Torretta ${ }^{1,3} \&$ Sandra S. Aliscioni ${ }^{1,2,3}$ \\ ${ }^{1}$ Cátedra de Botánica General, Facultad de Agronomía, Universidad de Buenos Aires, Av. San Martín 4453, C1417DSE, Buenos Aires, Argentina \\ ${ }^{2}$ Instituto de Botánica Darwinion (IBODA), Casilla de Correo 22, B1642HYD San Isidro, Buenos Aires, Argentina \\ ${ }^{3}$ Consejo Nacional de Investigaciones Científicas y Técnicas, Argentina \\ gomiz@agro.uba.ar; torretta@agro.uba.ar; saliscioni@darwin.edu.ar
}

\begin{abstract}
Resumen
Gómiz, N.E., Torretta, J.P. \& Aliscioni, S.S. 2014. Zygostates alleniana (Orchidaceae: Epidendroideae: Cymbidieae: Oncidiinae): estructura floral relacionada con la polinización. Anales Jard. Bot. Madrid 71(1): e002.

El género Zygostates Lindl. (Orchidaceae) comprende aproximadamente 20 especies de pequeñas plantas epífitas con distribución neotropical, representado en su límite más austral por la especie $Z$. alleniana. En el presente trabajo se estudian morfológica y anatómicamente las características florales de esta especie relacionadas con el mecanismo de polinización. Se confirma la presencia de tricomas unicelulares en la base del labelo y lóbulos laterales que actúan secretando aceite, constituyendo un elaióforo tricomatoso. El aceite se deposita por debajo de la cutícula en el ápice de los tricomas formando pequeñas ampollas. El aceite podría representar una recompensa para la especie Lophopedia nigrispinis, la cual sería un posible polinizador de $Z$. alleniana en un área natural dentro del rango de distribución geográfica de esta especie vegetal. Por otro lado, se comprueba que la reconfiguración del polinario se debe a la deshidratación de las paredes celulares. Esta reconfiguración podría favorecer la polinización cruzada, mecanismo ya descrito para otras especies de la familia Orchidaceae. Por último, se discuten los caracteres florales presentes en $Z$. alleniana con otras especies estrechamente emparentadas.
\end{abstract}

Palabras clave: aceites florales, abejas colectoras de aceite, Argentina, callo, estípite, recompensa, visitante floral, polinización, elaióforo.

\section{INTRODUCCIÓN}

El género Zygostates Lindl. (Orchidaceae), en su circunscripción actual, pertenece a la subfamilia Epidendroideae, tribu Cymbidieae, subtribu Oncidiinae (Chase \& al., 2003; Neubig \& al., 2012), y comprende aproximadamente 20 especies de pequeñas plantas epífitas con distribución neotropical, siendo especialmente rico en abundancia específica en el SE de Brasil (Toscano de Brito, 2001; Chase \& Toscano de Brito, 2009). En su límite más austral este género llega hasta Argentina, donde se encuentra representado únicamente por la especie Z. alleniana Kraenzl. citada para las provincias de Chaco, Corrientes, Formosa y Misiones (Williams, 1939; Johnson, 2001).

Originalmente, Zygostates formaba parte de la subtribu Ornithocephalinae, la cual incluía 15 géneros y casi 80 especies (Dressler, 1993; Toscano de Brito \& Kollman, 1997). Sobre la base de evidencia molecular se ha confirmado que estos géneros se reúnen en un grupo monofilético ("clado Ornithocephalus") dentro de la subtribu Oncidiinae, a la cual han sido transferidos recientemente (Neubig \& al., 2012). Estos géneros se caracterizan por presentar una morfología floral altamente compleja (Toscano de Brito, 2001).

\begin{abstract}
Gómiz, N.E., Torretta, J.P. \& Aliscioni, S.S. 2014. Zygostates alleniana (Orchidaceae: Epidendroideae: Cymbidieae: Oncidinae): Floral structure related to pollination. Anales Jard. Bot. Madrid 71(1): e002.

The genus Zygostates Lindl. (Orchidaceae) comprises about 20 species of small Neotropical epiphytic plants, represented in its southernmost limit by the species $Z$. alleniana. In this paper, we studied morphological and anatomical floral characteristics of this species related to pollination mechanism. We confirmed the presence of the unicellular trichomes on the base of the lip and side lobes secreting oil, constituting a trichomal elaiophore. The oil is deposited beneath the cuticle at the apex of the trichomes forming small blisters. The oil could represent a reward for the species Lophopedia nigrispinis, which would be a potential pollinator of $Z$. alleniana in a natural area within the geographic range of this plant species. Moreover, we prove that the reconfiguration of the pollinaruim is due to the dehydration of the walls cell. This reconfiguration could favor cross-pollination mechanism already described for other species of the family Orchidaceae. Finally, we discuss the floral characters present in $Z$. alleniana with closely related species.
\end{abstract}

Keywords: floral oils, oil-collecting bees, Argentina, callus, stipite, rewards, floral visitors, pollination, elaiophore.

La subtribu Onciidinae es una de las más variables en relación a la morfología floral, biología reproductiva y relaciones con los visitantes florales (Dressler, 1993). Si bien la mayoría de sus especies no ofrecen recompensas (Chase \& al., 2009), algunas secretan aceites en elaióforos florales. Dentro del "clado Ornithocephalus" se reportó la presencia de secreción de aceites florales en todos sus géneros (Renner \& Schaefer, 2010), aunque solo se describió la estructura del elaióforos en especies de los géneros Ornithocephalus Hook., Phymatidium Lindl. y Zygostates (Vogel, 1974; Toscano de Brito, 2001; Reis \& al., 2006; Pacek \& Stpiczyńska, 2007; Pacek \& al., 2012).

En particular para Zygostates, Buchmann (1987) confirma que cuatro de sus especies presentan elaióforos, aunque no las menciona ni describe la estructura de estas glándulas. Por otro lado, se han descrito elaióforos tricomáticos en el callo del lóbulo central en Z. pustulata (Kraenzl.) Schltr. (Vogel, 1974), Z. multiflora (Rolfe) Schltr. (Toscano de Brito, 2001), Z. grandiflora (Lindl.) Mansf. y Z. lunata Lindl. (Pacek \& al., 2012). Además, Toscano de Brito (2001) muestra la presencia de elaióforos sobre la superficie adaxial de los pétalos en $Z$. ovatipetala (Brade) Toscano.

* Autor para correspondencia. 
Las especies del "clado Ornithocephalus" poseen, en general, diminutas flores blancas, verdes o amarillentas, y Vogel (1974) sugiere que son visitadas por pequeñas abejas colectoras de aceites del género Paratetrapedia s.l., tribu Tapinotaspidini, familia Apidae. Sin embargo, existen pocas observaciones de campo que reportan las especies que participan de dicha interacción, van der Pijl \& Dodson (1969) mencionan visitas de Paratetrapedia testacea en flores de Ornithocephalus ciliatus Lindl. (citado como O. avicula) y O. cf. patentilobus C. Schweinf. y Paratetrapedia calcarata sobre flores de Ornithocephalus bicornis Lindl. ex Benth. y O. powellii Schltr.

La presencia de un callo tricomático en la base del labelo con actividad glandular es un carácter frecuente en el género Zygostates, pero la secreción de aceites no fue comprobada en todas sus especies. Asimismo, otras partes de la flor como la superficie adaxial de los pétalos pueden presentar actividad secretora (Toscano de Brito, 2001). En el presente trabajo, se estudian las características morfológicas y anatómicas de las flores de $Z$. alleniana asociadas a la polinización. Los objetivos son detectar la presencia de elaióforos, determinar su posición en la flor, conocer su estructura externa e interna y analizar como se produce la actividad secretora; también se pretende conocer los visitantes florales que interactúan con esta especie vegetal en un área natural dentro del rango de su distribución geográfica y analizar características estructurales de las flores, en particular a nivel del polinario y su posible consecuencia en el mecanismo de polinización. Por último, se comparan los caracteres florales presentes en $Z$. alleniana con otras especies estrechamente emparentadas y se plantean posibles tendencias evolutivas dentro de un contexto filogenético.

\section{MATERIAL Y MÉTODOS}

Durante el mes de octubre de 2011, se localizaron poblaciones naturales de $Z$. alleniana en diferentes localidades de la Provincia de Misiones, Argentina (Montecarlo, Depto. Montecarlo y alrededores del Parque Nacional Iguazú, Depto. Iguazú). Se realizaron observaciones de visitantes florales durante dos períodos diarios, 9-11 am y 14-16 pm de los días 11 y 12 de octubre. Conjuntamente también se capturaron visitantes florales que se encontraban interactuando con otras especies vegetales cercanas a los individuos de $Z$. alleniana, pertenecientes a otras familias que también ofrecen aceites como recompensa (Iridaceae y Malpighiaceae). Los ejemplares capturados fueron determinados en laboratorio, consultando bibliografía de referencia (Aguiar, 2009) y cotejando con ejemplares depositados en la colección entomológica de la Cátedra de Botánica General, Facultad de Agronomía, Universidad de Buenos Aires.

Algunas flores frescas maduras de Z. alleniana fueron fijadas en FAA (alcohol etílico 70\%, formol, ácido acético glacial; 90:5:5), para luego ser analizadas en laboratorio bajo lupa binocular y esquematizadas mediante el uso de cámara clara adosada a lupa binocular. La posición de los elaióforos se determinó en el campo sumergiendo flores frescas en una solución alcohólica saturada de Sudán III para detectar la presencia de lípidos (D’Ambrogio de Argüeso, 1986).

Un ejemplar de referencia fue depositado en el Herbario "G. Xuarez", Facultad de Agronomía, Universidad de Buenos Aires, (BAA 27033). También se estudiaron otros ejemplares de Z. alleniana depositados en los herbarios BAA y SI (Instituto de Botánica Darwinion) para comparar las características estructurales analizadas en el material fresco.

Para el estudio morfológico de los polinarios, se observaron con lupa binocular y dibujaron con cámara clara los polinarios extraídos de las flores fijadas, de los ejemplares de herbario y los adheridos a los visitantes florales capturados. En los dos últimos casos, los polinarios secos fueron restaurados usando solución de amonio concentrado según método descrito por Toscano de Brito (1996). De esta manera, se comparó la morfología de polinarios frescos, deshidratados y rehidratados (reconfigurados). Asimismo, se realizaron cortes transversales a mano alzada de polinarios a diferentes niveles, en particular a la altura del estípite, los cuales fueron teñidos con solución alcohólica de Sudán III y solución acuosa de Rojo de rutenio para detectar lípidos y sustancias pécticas respectivamente en las paredes celulares (D’Ambrogio de Argüeso, 1986). Algunos de los cortes, fueron montados en agua destilada y observados de manera continua bajo microscopio óptico por un período de aproximadamente 10 minutos hasta la deshidratación del material, con el objetivo de detectar cambios estructurales entre el material fresco y deshidratado.

Para los estudios anatómicos se utilizaron flores fijadas, a las cuales se cortó transversal y longitudinalmente previa inclusión en parafina siguiendo el método tradicional para histología vegetal mediante microtomo rotativo. Se obtuvieron cortes de 6-8 $\mu \mathrm{m}$ de espesor, se tiñeron con safranina-fast green y se montaron en medio de montaje sintético (Johansen, 1940). Para la detección de sustancias cristalinas, los mismos cortes fueron observados bajo luz polarizada. Todas las observaciones se realizaron con un microscopio óptico Motic (B1, Advances series) y fueron documentadas mediante fotomicrografías obtenidas con cámara digital adosada.

Para los estudios de microscopía electrónica de barrido (MEB), se tomó una flor completa, se la deshidrató mediante una serie ascendente de alcoholes etílicos hasta la suspensión en alcohol $100 \%$, para luego ser sometida a punto crítico usando $\mathrm{CO}_{2}$; posteriormente se metalizó la muestra y se examinó con un microscopio Philips XL 30 (O’Brien \& McCully, 1981).

\section{RESULTADOS}

\section{Morfología floral}

Las flores de $Z$. alleniana se reúnen en inflorescencias racemosas en número de $2-12$, de $2-3 \mathrm{~cm}$ de largo, sobrepasando a las hojas en longitud. Las flores son resupinadas, simétricas, largamente pediceladas, pequeñas, de 6,5-7,5 mm de diámetro, con sépalos y pétalos blancos que permanecen de esa coloración en la flor madura. Los pétalos son algo espatulados, de ápice redondeado, margen denticulado y superficie adaxial recubierta de pequeñas papilas.

El labelo está formado por tres lóbulos, los laterales son pequeños y tricomatosos, y el central, grande, replegado de forma navicular, blanco con base verdosa, carnoso, de margen grueso, con callosidad tricomatosa en la base que se angosta. El área tricomatosa se extiende desde un lóbulo lateral hacia el otro pasando por la base del labelo con un área aproximada de 2,2-2,4 ×0,7-0,9 mm. A cada lado de la columna se extiende un brazo ascendente de color verde claro y de superficie papilosa (Fig. 1). 


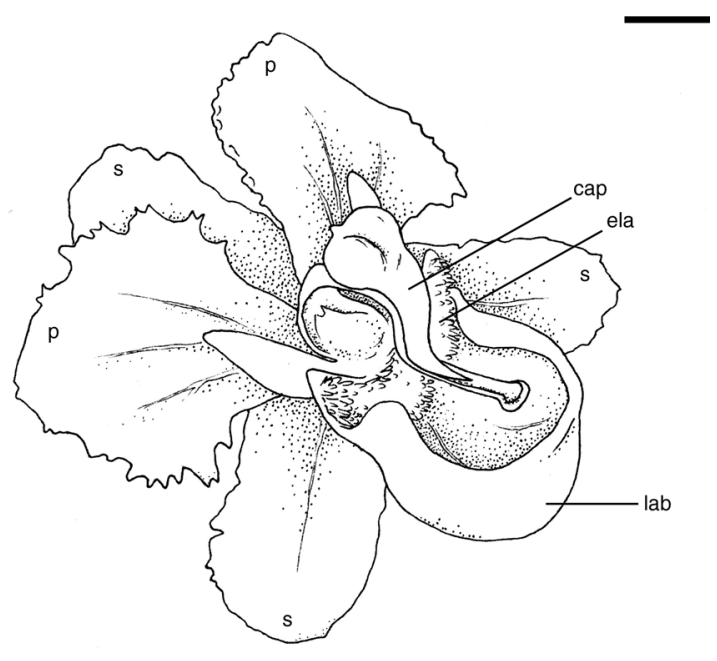

Fig. 1. Flor de Zygostates alleniana, en vista semilateral. cap, capuchón de la antera; ela, elaióforo; lab, labelo; $p$, pétalos; s, sépalos. Escala $=1 \mathrm{~mm}$.

La columna es esbelta, incurvada, de superficie lisa, se extiende por encima del labelo a modo de tobogán sosteniendo al polinario, que presenta una forma similar. La antera es bibulbosa en su base y largamente atenuada sobre el rostelo. La cavidad estigmática es cóncava, papilosa y está ubicada en la base de la columna. El polinario posee cuatro polinias distribuidas en dos pares iguales, superpuestos, con el estípite largo, sinuoso cuando fresco. El viscidio es pequeño, elíptico (Figs. 1, 2).

\section{Observaciones de campo}

Durante las observaciones de campo no se registraron visitantes florales interactuando con flores de Z. alleniana; sin embargo se capturaron cuatro hembras de Lophopedia nigrispinis sobre flores de Trimezia spathata (Klatt) Baker (familia Iridaceae). Estos individuos se encontraban raspando con sus patas delanteras la zona de los tépalos internos donde se encuentran tricomas glandulares secretores de aceites (Lovo \& al., 2012). Dos de las hembras capturadas presentaban 1 y 2 polinarios respectivamente adheridos

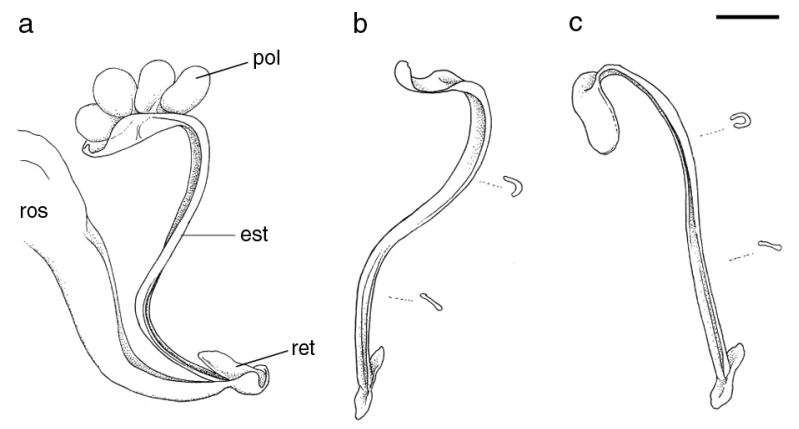

Fig. 2. Detalle del polinario y la columna de Zygostates alleniana. a, polinario parcialmente desprendido de la columna (sin el capuchón de la antera); b, c, estípite sin las polinias, indicando la forma del transcorte en dos diferentes posiciones para notar el arqueamiento diferencial del estípite (b, hidratado; $c$, deshidratado). pol, polinias; est, estípite; vis, viscidio; ros, rostelo. Escala $=1 \mathrm{~mm}$.

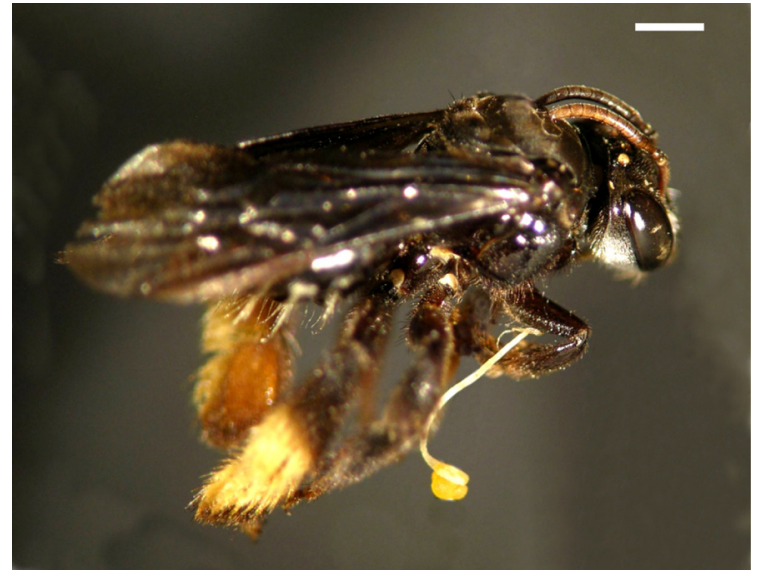

Fig. 3. Hembra de Lophopedia nigrispinis con un polinario de Zygostates alleniana adherido en la pata delantera. Escala $=1 \mathrm{~mm}$.

en sus tibias delanteras (Fig. 3). Los polinarios hallados fueron analizados bajo lupa y coinciden en su morfología con los de Z. alleniana.

A pesar de que Toscano de Brito (2001) menciona que algunas especies de Zygostates son fragantes, las flores de $Z$. alleniana analizadas en este estudio carecieron de fragancia perceptible.

\section{Reacción histoquimica sobre flores frescas}

La reacción con Sudán III sobre flores frescas resultó solamente positiva en el área tricomatosa (base del labelo y lóbulos laterales); los tricomas que son originalmente verdosos, adquirieron una coloración rojo intenso evidenciando la presencia de tejido secretor de aceites (Fig. 4). Asimismo, parte del polinario, en particular el estípite y el viscidio, también se tiñeron de rojo pero con menor intensidad; en este caso la reacción identifica el origen lipídico de la cutícula de las paredes celulares que forman estas estructuras.

\section{Microscopia óptica}

La zona tricomatosa se observa en la base del lóbulo central y lóbulos laterales del labelo; visto en corte longitudinal dicha zona queda cubierta parcialmente por la columna (Fig. 5a); en el corte transversal, esta zona es cóncava con una quilla media ligeramente elevada (Fig. 5c). La epidermis aquí está constituida íntegramente por tricomas unicelulares (Fig. 6a). Estos tricomas son cilíndricos, en algunos casos capitados, de 250-300 $\mu \mathrm{m}$ de largo por 20-25 $\mu \mathrm{m}$ de diámetro, con citoplasma denso y pared celular delgada. En la mayoría de los tricomas, se observa que la cutícula en la zona del ápice de los mismos se distiende formando una pequeña ampolla (de 35-40 um de diámetro) más ancha que el diámetro de los tricomas (Fig. 6b). La cutícula tiene un espesor de 1-1,5 $\mu \mathrm{m}$ en las paredes laterales de los tricomas, siendo más gruesa sobre la ampolla donde llega a 7-8,5 $\mu \mathrm{m}$ de espesor. El citoplasma se encuentra periféricamente y gran parte del volumen celular es ocupado por una gran vacuola central. Los núcleos se observan densos, esféricos o alargados acompañando la forma del tricoma, de 7-8 ×11-17 $\mu \mathrm{m}$, ubicados en la mayoría de los casos, en la mitad inferior del tricoma y presentando notorios nucléolos. Por debajo de la 

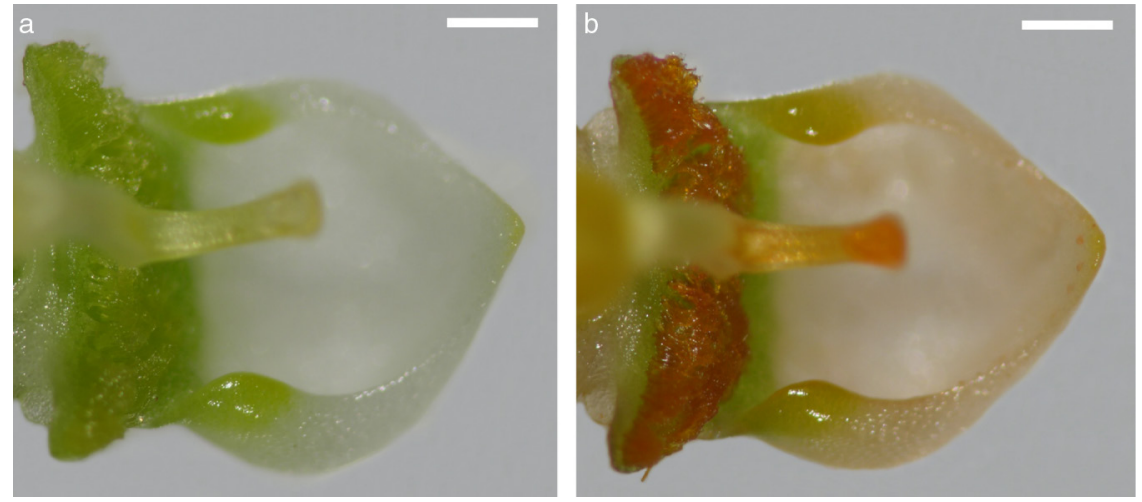

Fig. 4. Detalle de labelo y columna de flores frescas de Zygostates alleniana. a, coloración natural; b, con tinción de Sudán III. Escalas=0,5 mm.
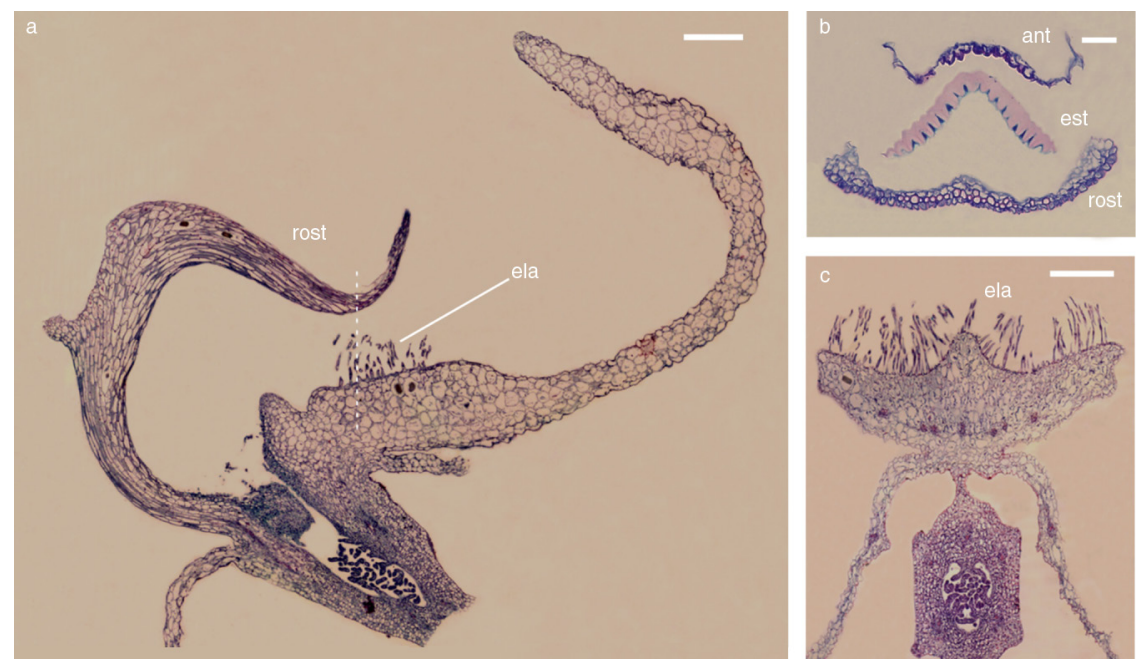

Fig. 5. Fotomicrografías de flores de Zygostates alleniana vistas al microscopio óptico. a, corte longitudinal (se han retirado el estípite con las polinias y el capuchón de la antera); $\mathbf{b}, \mathbf{c}$, cortes transversales a nivel de la línea punteada indicada en a; $\mathbf{b}$, detalle de rostelo, estípite y capuchón de la antera; c, detalle de la zona tricomatosa en la base del labelo; ant, caperuza de la antera; ela, elaióforo; est, estípite; rost, rostelo. Escalas: a, c=300 $\mu$ m; $\mathrm{b}=50 \mu \mathrm{m}$.

epidermis tricomatosa, se encuentra un parénquima subyacente compacto, con células grandes, isodiamétricas, incoloras, con grandes vacuolas centrales, con escasos cloroplastos y núcleos de posición periférica. En esta zona parenquimática, se observan grandes idioblastos conteniendo rafidios que fueron confirmados mediante luz polarizada (Fig. 6c). El labelo se encuentra vascularizado con varios haces colaterales, la epidermis inferior esta formada por células planas a ligeramente papilosas.

En vista transversal de la columna, a la altura de la base del labelo, se observa en detalle el capuchón de la antera, el estípite del polinario y el rostelo. El estípite del polinario está constituido por paredes celulares muy engrosadas con cutícula desarrollada (Fig. 5b). Las paredes de cada célula toman aspecto de "U" invertida hacia el rostelo (Fig. 7b). Las observaciones realizadas a la altura del viscidio denotan que la capa celular que da origen al estípite proviene de la superficie más externa del rostelo, que posteriormente se desprende quedando las paredes celulares radiales y tangenciales externas como parte del estípite, mientras que la pared tangencial interna permanece unida al rostelo. La tinción con rojo de rutenio realizada sobre cortes transversales a mano alzada del estípite indica que las células presentan paredes primarias con gran contenido de pectatos (Fig. 7a), y la tinción con Sudán III resultó positiva, evidenciando el origen lipídico de las gruesas cutículas que cubren dichas paredes (Fig. 7c, d).

\section{Microscopía electrónica de barrido}

Mediante el uso de microscopía electrónica de barrido pudo observarse que los tricomas secretores difieren en longitud y diámetro (Fig. 8a), algunos son de forma cilíndrica con un diámetro semejante en toda su longitud, con ápice de forma redondeada y superficie lisa (Fig. 8b), mientras que otros presentan el ápice ligeramente ensanchado dándoles un aspecto capitado. La cutícula de los mismos se observa de lisa a escasamente granulosa, pero no se registran poros o rupturas de la misma para la liberación de los aceites acumulados. Las células epidérmicas que delimitan 

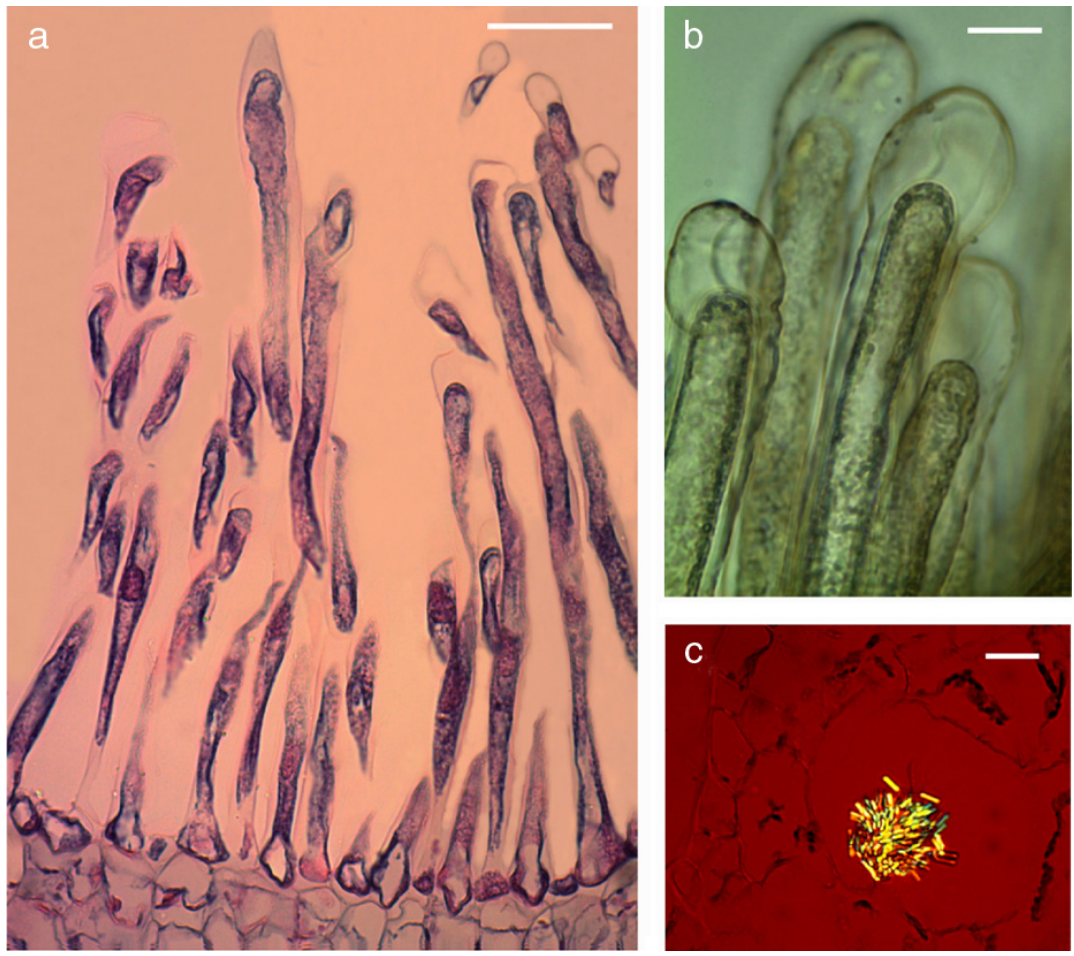

Fig. 6. Fotomicrografías del elaióforo de Zygostates alleniana vistas al microscopio óptico. a, tricomas unicelulares; b, detalle de ápices de tricomas con ampolla cuticular teñido con Sudán III; c, idioblasto con ráfides en el parénquima subepidérmico visto con luz polarizada. Escalas: a, c=50 $\mu \mathrm{m} ; \mathrm{b}=20 \mu \mathrm{m}$.
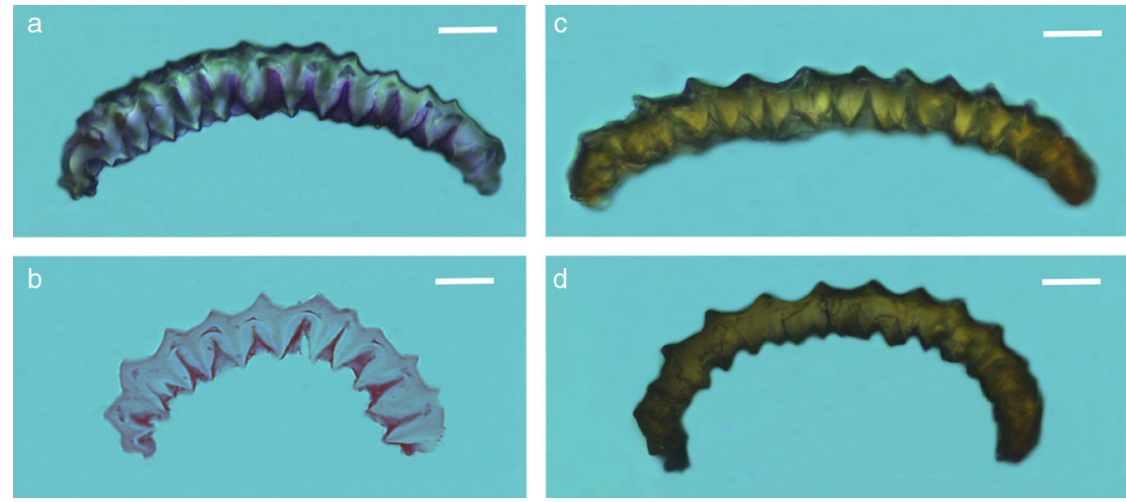

Fig. 7. Fotomicrografías de cortes transversales de estípites de Zygostates alleniana vistos al microscopio óptico (a, c, hidratados; b, d, deshidratados). a, con rojo de rutenio; b, con safranina-fast green; $\mathbf{c}$, d, con Sudán III. Escalas = $30 \mu \mathrm{m}$.

la zona tricomatosa son de paredes rectas a escasamente arqueadas (Fig. 8a).

\section{Observaciones sobre la reconfiguración del polinario}

Las observaciones bajo lupa binocular revelaron que el polinario de $Z$. alleniana presenta una configuración característica de aspecto sinuoso, cuando el material está fresco recién extraído de la flor o fijado en líquidos conservantes. Una vez retirado de la flor, permanece de igual forma un breve período de tiempo; alrededor de 1 minuto mientras se encuentra hidratado. A medida que se va deshidratando cambia su configuración original, tomando forma de coma progresivamente, proceso que demora 5-7 minutos. Los polinarios extraídos de los ejemplares de herbario de Z. alleniana, y aquellos adheridos a las patas de los individuos de Lophopedia nigrispinis capturados, se encontraban reconfigurados (Figs. 2c, 3); pero al tratarlos con solución de amonio recobraron la forma sinuosa original (Fig. 2b).

\section{DISCUSIÓN}

En este trabajo se confirma la presencia de elaióforos florales en $Z$. alleniana cuyos caracteres estructurales coinciden en líneas generales con los descritos para otras especies del género (Vogel, 1974; Toscano de Brito, 2001; Pacek \& Stpiczyńska, 2007; Pacek \& al., 2012). Los elaióforos de Z. alleniana están formados por tricomas unicelulares, 

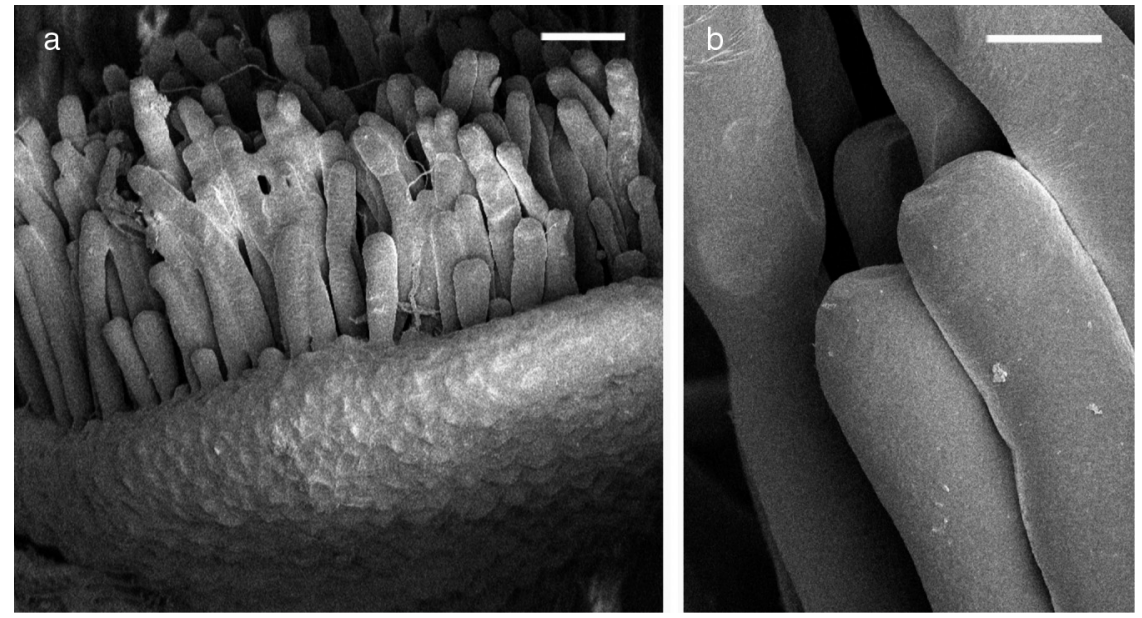

Fig. 8. Fotomicrografías del elaióforo de Zygostates alleniana vistas al microscopio electrónico de barrido. a, área tricomatosa sobre el lóbulo lateral del labelo; b, detalle de los tricomas. Escalas: $a=50 \mu \mathrm{m} ; b=10 \mu \mathrm{m}$.

localizados en la base del lóbulo central y en los lóbulos laterales del labelo. A diferencia de lo descrito para Z. ovatipetala (Toscano de Brito, 2001), en la especie aquí estudiada no se detectó actividad secretora en la superficie adaxial de los pétalos. El elaióforo consta de una capa de tejido epidérmico representado por tricomas unicelulares, con citoplasmas densos y núcleos relativamente grandes, lo que evidencia una importante actividad metabólica celular. Por debajo, se dispone un tejido parenquimático subepidérmico, que Pacek \& al. (2012) describen como sub-secretor ya que acompañaría al proceso de secreción, por lo que frecuentemente contiene almidón y/o lípidos además de numerosos idioblastos con rafidios. En el caso de Z. alleniana se observó dicho tejido con rafidios, aunque sin almidón ni gotas lipídicas, y solo conteniendo escasos cloroplastos. Existen diversos reportes de rafidios asociados a elaióforos para la subtribu Oncidiinae (Stpiczyńska \& al., 2007; Stpiczyńska \& Davies, 2008; Pacek \& Stpiczyńska, 2007; Aliscioni \& al., 2009; Gómiz \& al., 2013). Sandoval-Zapotitla \& al. (2010) muestran que estos cristales son los más frecuentes en esta subtribu, inclusive en órganos vegetativos como las hojas; esto indicaría que los rafidios podrían no estar asociados exclusivamente a estructuras secretoras.

Para la subtribu Oncidiinae, la secreción del aceite en elaióforos fue descrita mediante distensión de la cutícula formando una ampolla como en Gomesa paranaensis (Kraenzl.) M.W. Chase \& N.H. Williams (Singer \& Cocucci, 1999), G. radicans (Rchb. f.) M.W. Chase \& N.H. Williams (Stpiczyńska \& Davies, 2008), Oncidium cheirophorum Rchb. f., Ornithocephalus kruegeri Rchb. f. (Pacek \& Stpiczyńska, 2007), Trichocentrum cavendishianum (Bateman) M.W. Chase \& N.H. Williams (Stpiczyńska \& al., 2007), Oncidium ornithorbynchum Kunth (Davies \& Stpiczynska, 2009), Gomesa echinata (Barb. Rodr.) M.W. Chase \& N.H. Williams, G. ranifera (Lindl.) M.W. Chase \& N.H. Williams, Oncidium amazonicum (Schltr.) M.W. Chase \& N.H. Williams, O. oxyceras (Königer \& J.G. Weinm.) M.W. Chase \& N.H. Williams (Stpiczyńska \& al., 2013), Ornithocephalus gladiatus Hook, Phymatidium falcifolium Lindl., Zygostates grandiflora, Z. lunata (Pacek \& al., 2012), o por pasaje a través de la pared depositándose sobre ella como en Gomesa bifolia (Sims)
M.W. Chase \& N.H. Williams (Aliscioni \& al., 2009), G. loefgrenii (Cogn.) M.W. Chase \& N.H. Williams, G. recurva R. Br. (Stpiczyńska \& al., 2007), G. venusta (Drapiez) M.W. Chase \& N.H. Williams (Stpiczyńska \& Davies, 2008), G. flexuosa (Lodd.) M.W. Chase \& N.H. Williams, G. riograndensis (Cogn.) M.W. Chase \& N.H. Williams, G. varicosa (Lindl.) M.W. Chase \& N.H. Williams. (Gómiz \& al., 2013), y Rudolfiella picta (Schltr.) Hoehne (Davies \& Stpiczyńska, 2009). Sería necesario realizar estudios ontogenéticos en especies con distintos mecanismos de secreción para detectar si verdaderamente ambos procesos de secreción son excluyentes, ya que otra posibilidad sería que dependiera del grado de desarrollo de la flor y en particular del elaióforo.

Pacek \& al. (2012) diferencian a Phymatidium falcifolium de otras especies estudiadas del "clado Ornithocephalus" por presentar tricomas capitados, mientras que en las otras son no-capitados. Sin embargo, Toscano de Brito (2001) menciona la presencia de tricomas capitados y no-capitados en flores de un mismo individuo en esta especie. Este hecho podría deberse a la cantidad de aceite acumulado en el ápice del tricoma. En este trabajo se reporta la presencia de ambos tipos de tricomas en $Z$. alleniana.

Los aceites secretados por Z. alleniana serían colectados por hembras de Lophopedia nigrispinis. Los aceites florales, mezclados con polen de otras flores, podrían ser utilizados como un constituyente del alimento para las larvas (Simpson \& Neff, 1981; Simpson \& al., 1977; Machado, 2004), posiblemente también para los adultos (Buchmann, 1987). Para realizar la colecta, estas especies utilizan setas especializadas ubicadas en los basitarsos del primer par de patas (Roig Alsina, 1997; Aguiar, 2009). Durante el comportamiento de forrajeo, a las abejas se les adhiere el polinario en las tibias de las patas delanteras. Si bien individuos de L. nigrispinis no han sido observados directamente sobre flores de Z. alleniana, el hallazgo de polinarios pertenecientes a dicha especie adheridos al cuerpo de dos hembras, permite inferir que esta especie de abeja podría ser un potencial polinizador. Dado que no se ha podido observar directamente el polinizador sobre la flor, es posible plantear dos hipótesis de forrajeo (no excluyentes): a) que las abejas colecten el aceite floral posadas sobre la flor, la inflorescencia u otra parte de 
la planta, o bien, b) que las abejas utilicen alguna parte de la flor como tabula infraestigmatica, de la cual la abeja pueda sujetarse con las mandíbulas mientras utiliza las patas para la obtención de aceites. Es necesario realizar más observaciones de campo para confirmar la efectiva interacción entre L. nigrispinis y $Z$. alleniana, así de cómo se realiza el proceso de recolección del aceite.

En el Neotrópico, numerosas especies de abejas colectan aceites florales, principalmente de las tribus Centridini, Tapinotaspidini y Tetrapediini, todas de la familia Apidae (Michener, 2007). Por lo tanto, existen notables variaciones en las estructuras colectoras de aceites entre los diferentes géneros (Roig Alsina, 1997, 2000; Cocucci \& al., 2000). Algunos autores (Machado, 2004) comentan que la estructura del órgano colector del polinizador podría relacionarse directamente con diferentes tipos de elaióforos. Por ejemplo, la mayoría de especies de Centris y Epicharis (Centridini) generalmente presentan peines en el basitarso del primer y segundo par de patas, formados por filas de setas especializadas en la recolección de aceites; dicha estructura se considera principalmente asociada a elaióforos epiteliales, al menos para la familia Malpighiaceae (Vogel, 1974; Neff \& Simpson, 1981; Sazima \& Sazima, 1989).

Para la familia Orchidaceae, Pacek \& al. (2012) postulan que las especies que tienen elaióforos epiteliales son polinizadas por insectos de mayor tamaño que pueden sujetarse de la tabula infraestigmatica con sus mandíbulas y, con sus primer y segundo pares de patas, raspar los elaióforos que se disponen a pares lateralmente sobre el labelo; por otro lado, las especies con elaióforos tricomáticos que se encuentran centralmente localizados son visitadas por abejas pequeñas que raspan las flores de manera más delicada. Los resultados obtenidos para Z. alleniana soportan esta hipótesis, aunque observaciones de campo en zonas cercanas al área de estudio, revelaron la presencia de Lophopedia nigrispinis interactuando con flores de tres especies de Malpighiaceae (Torretta, observación personal), familia que presenta elaióforos epiteliales. Otras especies de Paratetrapedia s.l. también visitan especies de Malpighiaceae (Sazima \& Sazima, 1989; Aguiar, 2009). De la misma manera, la relación entre la morfología de la abeja y el modo de forrajeo no estaría estrictamente relacionado con el tipo de elaióforos, como ha sido demostrado en diferentes familias de plantas en las que se registró visitas de Centris y que poseen elaióforos tricomáticos (Neff \& Simpson, 1981; Vogel, 1988; Vogel \& Machado, 1991; Cocucci, 1991; Vogel \& Cocucci, 1995; Sérsic \& Cocucci, 1999; Machado \& al., 2002).

En relación con la estructura del polinario, existen evidencias de que su reconfiguración favorece la polinización cruzada, ya que el cambio de forma del estípite es más lento que el tiempo entre visitas a diferentes flores de la misma planta (Darwin, 1862; Peter \& Johnson; 2005). Una vez reconfigurado el polinario, la inserción de las polinias en la superficie estigmática se favorece. Johnson \& al. (2004), confirmaron que la polinización cruzada en Anacamptis morio (L.) R.M. Bateman \& M.W. Pridgeon Chase tendría lugar si el tiempo de reconfiguración del polinario superase al tiempo de visita de los polinizadores. Darwin (1862) menciona para diversas especies de los géneros Maxillaria Ruiz \& Pav., Odontoglossum Kunth, Oncidium Sw. s.l., Orchis Tourn., entre otros, que los polinarios están erectos al ser extraídos y después de un tiempo se reconfiguran.
Este mecanismo impediría la autopolinización ya que, si el polinizador insiste en la misma flor o visita una muy próxima, el ángulo en que se encontrarían todavía las polinias no le permitiría contactar con la superficie estigmática. Al cabo de un tiempo variable se produce la modificación del polinario, de manera que podría fácilmente entrar en contacto con la superficie estigmática (Singer, 2009).

El mecanismo de reconfiguración del polinario se mantiene en la actualidad escasamente documentado, pero algunos trabajos preliminares (Endress, 1998) soportan la teoría de Darwin (1862) de que la reconfiguración en algunas orquídeas involucra la deshidratación de diferentes capas de tejidos de estructuras accesorias de los polinarios. Darwin notó que el viscidio y la base del estípite pueden ser importantes en causar la reconfiguración (Peter \& Johnson, 2006).

En Z. alleniana el estípite está formado por una capa de células epidérmicas con paredes primarias ricas en pectatos diferencialmente engrosadas y gruesas cutículas, las cuales al deshidratarse permitirían que el polinario tenga un movimiento de acordeón. Del lado de la columna, al ser las estriaciones más pronunciadas que del lado del capuchón de la antera, el acortamiento es mayor, lo que provoca una concavidad hacia ese lado. La parte media del estípite es la región que presenta un mayor plegamiento, de lo que resulta una reconfiguración de todo el polinario y una diferente orientación de las polinias y el viscidio.

Diversos estudios han demostrado que dentro de la subtribu Oncidiinae los elaióforos epiteliales son los más comunes (Singer \& Cocucci, 1999; Reis \& al., 2006; Stpiczyńska \& al., 2007; Stpiczyńska \& Davies, 2008; Pacek \& Stpiczyńska, 2007; Aliscioni \& al., 2009; Pansarin \& Pansarin, 2010, Pacek \& al., 2012; Gómiz \& al., 2013); en menor medida se registraron elaióforos tricomáticos para los géneros Lockbartia, Ornithocephalus, Phymatidium y Zygostates (Vogel, 1974; Buchmann, 1987; Toscano de Brito, 2001; Reis \& al., 2006; Pacek \& Stpiczyńska, 2007; Pacek \& al., 2012, Blanco \& al., 2013). Pacek \& al. (2012) estudiaron los elaióforos de cuatro especies del "clado Ortinocephalus", y distinguen a O. gladiatus por presentar elaióforos del tipo intermedio dispuestos lateralmente, mientras que Phymatidium falcifolium, Zygostates grandiflora y Z. lunata los presentan del tipo tricomáticoso, ubicados centralmente sobre el callo del labelo. Elaióforos que combinan una parte epitelial y otra tricomatosa en la tribu Oncidiinae, se describieron en Gomesa flexuosa (Gómiz \& al., 2013), y en otros grupos de Orchidaceae, como en la especie Grobya amberstiae Lindl. de la subtribu Catasetinae (Mickeliunas-Pansarin \& al., 2009).

Dentro de Oncidiinae hay especies que poseen flores miméticas a las de Malpighiaceae (Powell \& et al., 2003; Vale \& al., 2011; Papadopulos \& al., 2013), las cuales carecen de elaióforos y atraen a los visitantes por engaño. Otras especies, cuyas flores también son semejantes a las Malpighiaceae, poseen elaióforos y ofrecen recompensa a sus polinizadores. Estos dos grupos de Oncidiinae (productoras de aceites vs. deceptivas) serían más complejos de analizar dentro de un contexto evolutivo, ya que el desarrollo de los elaióforos podría depender en mayor medida del medio y de las interacciones con otras especies. En un trabajo reciente sobre elaióforos en los géneros Gomesa y Oncidium, Stpiczynska \& al. (2013) concluyen que muchos caracteres de los elaióforos son compartidos por diferentes clados de la subtribu Oncidiinae, por lo cual serían homoplásticos y tendrían un valor limitado 
para analizarlos evolutivamente. Contrariamente, las especies del "clado Ornithocephalus", que en general presentan flores pequeñas y blanco-verdosas, atraen a abejas pequeñas colectoras de aceites que son sus polinizadores naturales. En este grupo monofilético de especies (Neubig \& al., 2012), la presencia de aceites florales es indispensable para que se lleve a cabo la polinización, y el mimetismo no ha evolucionado como estrategia dentro del grupo como lo ha hecho en otros clados de Oncidiinae.

Renner \& Schaefer (2010) recopilan la información disponible sobre géneros que presentan aceites florales; y en particular para el "clado Ornithocephalus" mencionan a todos los géneros como conteniendo especies secretoras de aceites. Sin embargo, no se sabe cuántas especies de cada género ofrecen aceite, cómo es la estructura del elaióforo o dónde se ubica.

La filogenia de la subtribu Oncidiinae publicada por Neubig \& al. (2012) contiene seis especies de Zygostates, incluyendo la especie aquí estudiada, y demuestra que el género es monofilético. Asimismo, todas las especies del "clado Ornithocephalus" analizadas en el mencionado trabajo, también se reúnen en un grupo monofilético con alto soporte. Por lo tanto, es probable que la presencia de elaióforos haya surgido en el ancestro común representando una sinapomorfía para este grupo. Se ha avanzado mucho sobre el conocimiento del "clado Ornithocephalus" y su posición filogenética dentro de la subtribu Oncidiinae, sin embargo es necesario continuar los estudios anatómicos y morfológicos sobre la diversidad floral, ampliar las hipótesis filogenéticas y acrecentar los trabajos de campo que registren información sobre las relaciones específicas entre planta y polinizador. Este último tema es el que aún deja numerosos interrogantes.

\section{AGRADECIMIENTOS}

A Gastón R. Sánchez, por su ayuda en el procesamiento de las imágenes; a Beatriz G. Galati y Gabriela E. Zarlavsky, por sus recomendaciones en los tratamientos histológicos; a Mariano Bello, por facilitar material vegetal, y a los revisores anónimos, por la lectura crítica del manuscrito. Los fondos para desarrollar este trabajo fueron provistos por la Universidad de Buenos Aires (UBACYT-20020090100068) y por el Consejo Nacional de Investigaciones Científicas y Técnicas (11220110100312). NEG es becaria UBACYT, y JPT y SSA son investigadores de CONICET, Argentina.

\section{REFERENCIAS BIBLIOGRÁFICAS}

Aguiar, A. 2009. Taxonomic revision of the bee genus Lophopedia Michener and Moure (Hymenoptera, Apidae, Tapinotaspidini). Zootaxa 2193: 1-52.

Aliscioni, S.S., Torretta, J.P., Bello, M. \& Galati, B.G. 2009. Elaiophores in Gomesa bifolia (Sims) M.W. Chase \& N.H. Williams (Oncidiinae: Cymbidieae: Orchidaceae): structure and oil secretion. Annals of Botany 104: 1141-1149. http://dx.doi.org/10.1093/ aob/mcp199.

Blanco, M.A., Davies, K.L., Stpiczynska, M., Carlsward, B.S., Ionta, G.M. \& Gerlach, G. 2013. Floral elaiophores in Lockhartia Hook. (Orchidaceae: Oncidiinae): their distribution, diversity and anatomy. Annals of Botany, http://dx.doi.org/10.1093/aob/ mct232.

Buchmann, S.L. 1987. The ecology of oil flowers and their bees. Annual Review of Ecology and Systematics 18: 343-369. http://dx. doi.org/10.1146/annurev.es.18.110187.002015.

Chase, M.W., Barrett, R.L., Cameron, K.N. \& Freudenstein, J.V. 2003. DNA data and Orchidaceae systematics: a new phylogenetic classification. En K.M. Dixon (ed.), Orchid conservation, pp. 69-89. Natural History Publications, Kota Kinabalu, Sabah.
Chase, M.W. \& Toscano de Brito, A.L.V. 2009. Zygostates. En: A.M. Pridgeon \& al. (eds.), Genera Orchidacearum, Volume 5: Epidendroideae, pp. 391-394. Oxford University Press, Oxford.

Chase, M.W., Williams, N.H., Faria, A.D., Neubig, K.M., Amaral, M.C.E. \& Whitten, W.M. 2009. Floral convergence in Oncidiinae (Cymbidieae; Orchidaceae): an expanded concept of Gomesa and a new genus Nobawilliamsia. Annals of Botany 104: 387-402. http:// dx.doi.org/10.1093/aob/mcp067.

Cocucci, A.A. 1991. Pollination biology of Nierembergia (Solanaceae). Plant Systematic and Evolution. 174: 17-35. http://dx.doi. org/10.1007/BF00937691.

Cocucci, A.A., Sérsic, A. \& Roig Alsina, A. 2000. Oil-collecting structures in Tapinotaspidini: their diversity, function and probable origin. Mitteilungen Muenchener Entomologischen Gesellschaft 90 51-74.

D’Ambrogio de Argüeso, A.C. 1986. Manual de técnicas en histología vegetal. Hemisferio Sur: Buenos Aires.

Darwin, C.R. 1862. On the various contrivances by which British and foreign orchids are fertilised by insects, and on the good effects of intercrossing. John Murray: London.

Davies, K.L. \& Stpiczyńska M. 2009. Comparative histology of floral elaiophores in the orchids Rudolfiella picta (Schltr.) Hoehne (Maxillariinae sensu lato) and Oncidium ornithorbynchum HBK (Oncidiinae sensu lato). Annals of Botany 104: 221-234. http://dx. doi.org/10.1093/aob/mcp119.

Dressler, R.L. 1993. Phylogeny and classification of the orchid family. Dioscorides Press: Oregon.

Endress, P.K. 1998. Diversity and evolutionary biology of tropical flowers. Cambridge University Press: Cambridge.

Gómiz, N.E., Torretta, J.P. \& Aliscioni, S.S. 2013. Comparative anatomy of elaiophores and oil secretion in the genus Gomesa (Orchidaceae). Turkish Journal of Botany 37: 859-871. http://dx. doi.org/10.3906/bot-1209-6.

Johansen, D.A. 1940, Plant Microtechnique. McGraw-Hill Book Company, Inc: New York.

Johnson, A.E. 2001. Las Orquídeas del Parque Nacional Iguazú. L.O.L.A.: Buenos Aires.

Johnson, S.D., Peter, C.I. \& Agren, J. 2004. The effects of nectar addition on pollen removal and geitonogamy in the non-rewarding orchid Anacamptis morio. Proceedings Royal Society B 271: 803-809.

Lovo, J., Winkworth, R.C. \& Mello-Silva, R. 2012. New insights into Trimezieae (Iridaceae) phylogeny: What do molecular data tell us? Annals of Botany 110: 689-702. http://dx.doi.org/10.1093/aob/ mcs127.

Machado, I.C. 2004. Oil-collecting bees and related plants: a review of the studies in the last twenty years and case histories of plants ocurring in NE Brazil. En: B.M. Freitas \& al. (eds.), Solitary bees. Conservation, rearing and management for pollination, pp. 255-280. Imprensa Universitária, Fortaleza.

Machado, I.C., Vogel, S. \& Lopes, A.V. 2002. Pollination of Angelonia cornigera Hook. (Scrophulariaceae) by long-legged, oil-collecting bees in NE Brazil. Plant Biology 4: 352-359. http://dx.doi.org/ $10.1055 / \mathrm{s}-2002-32325$.

Michener, C.D. 2007. The bees of the World. The Johns Hopkins University Press, Baltimore.

Mickeliunas-Pansarin, L., Morales-Castro M. \& Sazima, M. 2009. Osmophore and elaiophores of Grobya amberstiae (Catasetinae, Orchidaceae) and their relation to pollination. Botanical Journal of the Linnean Society 159: 408-415. http://dx.doi. org/10.1111/j.1095-8339.2009.00953.x.

Neff, J.L. \& Simpson, B.B. 1981. Oil-collecting structures in Anthophoridae (Hymenoptera): morphology, function, and use in systematics. Journal of the Kansas Entomological Society 54: 95-123.

Neubig, K.M., Whitten, W.M., Williams, N.H., Blanco, M.A., Endara L., Burleigh, J.G., Silveira, K., Cushman, J.C. \& Chase, M.W. 2012. Generic recircumscriptions of Oncidiinae (Orchidaceae: Cymbidieae) based on maximum likelihood analysis of combined DNA datasets. Botanical Journal of the Linnean Society 168 117-146. http://dx.doi.org/10.1111/j.1095-8339.2011.01194.x.

O’Brien T.P. \& McCully, M.E. 1981. The study of plant structure principles and selected methods. Termarcarphi Pty. Ltd. Melbourne.

Pacek, A. \& Stpiczyńska, M. 2007. The structure of elaiophores in Oncidium cheirophorum Rchb. f. and Ornithocephalus kruegeri Rchb. f. (Orchidaceae). Acta Agrobotanica 60: 9-14. http://dx.doi. org/10.5586/aa.2007.024.

Pacek, A., Stpiczyńska, M., Davies, K.L. \& Szymczak, G. 2012. Floral elaiophore structure in four representatives of the Ornithocephalus clade (Orchidaceae: Oncidiinae). Annals of Botany 110: 809-820. http://dx.doi.org/10.1093/aob/mcs158. 
Pansarin, E.M. \& Pansarin, L.M. 2011. Reproductive biology of Trichocentrum pumilum: an orchid pollinated by oil-collecting bees. Plant Biology 4: 576-581. http://dx.doi. org/10.1111/j.1438-8677.2010.00420.x.

Papadopulos, A.S.T., Powell, M.P., Pupulin, F., Warner, J., Hawkins, J.A., Salamin, N., Chittka, L., Williams, N.H., Whitten, W.M., Loader, D., Valente, L.M., Chase, M.W. \& Savolainen, V. 2013. Convergent evolution of floral signals underlies the success of Neotropical orchids. Proceedings of the Roval Society B 280: 20130960. http://dx.doi org/10.1098/rspb.2013.0960.

Peter, C.I. \& Johnson, S.D. 2006. Doing the twist: a test of Darwin's cross-pollination hypothesis for pollinarium reconfiguration. Biology Letters 2: 65-68. http://dx.doi.org/10.1098/ rsbl.2005.0385

Pijl, L. van der \& Dodson, C.H. 1969. Orchid flowers: their pollination and evolution. University of Miami Press: Coral Gables.

Powell, M.P., Pupulin, F., Warner, J., Chase, M.W. \& Savolainen, V. 2003. Floral mimicry in Oncidioid orchids. Lankesteriana 7: $109-110$.

Reis, M.G., Singer, R.B., Goncalves, R. \& Marsaioli, A.J. 2006. The chemical composition of Phymatidium delicatulum and P. tillandsioides (Orchidaceae) floral oils. Natural Product Communication 1: $757-761$.

Renner, S.S. \& Schaefer, H. 2010. The evolution and loss of oil-offering flowers: new insights from dated phylogenies for angiosperms and bees. Philosophical Transactions of the Royal Society B 365: 423-435. http://dx.doi.org/10.1098/rstb.2009.0229.

Roig Alsina, A. 1997. A generic study of the bees of the tribe Tapinotaspidini, with notes on the evolution of their oil-collecting structures (Hymenoptera, Apidae). Mitteilungen Muenchener Entomologischen Gesellschaft 87: 3-21.

Roig Alsina, A. 2000. Clave para las especies argentinas de Centris (Hymenoptera, Apidae), con descripción de nuevas especies y notas sobre distribución. Revista del Museo Argentino de Ciencias Naturales n.s. 2: 171-193.

Sandoval-Zapotitla, E., Terrazas, T. \& Villaseñor, J.L. 2010. Diversidad de inclusiones minerales en la subtribu Oncidiinae (Orchidaceae) Revista de Biología Tropical 58: 733-755.

Sazima, M. \& Sazima, I. 1989. Oil-gathering bees visit flowers of eglandular morphs of the oil-producing Malpighiaceae. Botanica Acta 102: 106-111. http://dx.doi.org/10.1111/j.1438-8677.1989. tb00073.x.

Sérsic, A.N. \& Cocucci, A.A. 1999. An unusual kind of nectary in the oil flowers of Monttea: its structure and function. Flora 194: 393-404.

Simpson, B.B. \& Neff, J.L. 1981. Floral rewards: alternatives to pollen and nectar. Annals of the Missouri Botanical Garden 68: 301-322. http://dx.doi.org/10.2307/2398800

Simpson, B.B., Neff, J.L. \& Seigler, D. 1977. Krameria, free fatty acids and oil-collecting bees. Nature 267: 150-151. http://dx.doi. org/10.1038/267150a0.
Singer, R.B. 2009. Morfología floral y polinización de orquídeas: el segundo libro de Charles Darwin. Acta Biológica Colombiana 14: 337-348.

Singer, R.B. \& Cocucci, A.A. 1999. Pollination mechanisms in four sympatric southern Brazilian Epidendroideae orchids. Lindleyana 14: 47-56.

Stpiczyńska, M., Davies, K.L., \& Gregg, A. 2007. Elaiophore diversity in three contrasting members of Oncidiinae (Orchidaceae). Botanical Journal of the Linnean Society 155: 135-148. http://dx.doi. org/10.1111/j.1095-8339.2007.00681.x.

Stpiczyńska, M. \& Davies, K. 2008. Elaiophore structure and oil secretion in flowers of Oncidium trulliferum Lindl. and Ornithophora radicans (Rchb. f.) Garay \& Pabst (Oncidiinae: Orchidaceae). Annals of Botany 101: 375-384. http://dx.doi.org/10.1093/aob/ $\mathrm{mcm} 297$.

Stpiczyńska, M., Davies, K.L., Pacek-Bieniek, A. \& Kamińska, M. 2013. Comparative anatomy of the floral elaiophore in representatives of the newly re-circumscribed Gomesa and Oncidium clades (Orchidaceae: Oncidiinae). Annals of Botany 112: 839-854. http:// dx.doi.org/10.1093/aob/mct149.

Toscano de Brito, A.L.V. 1996. The use of concentrated ammonia as an excellent medium for the restoration of orchid pollinaria: an example from the Ornithocephalinae (Orchidaceae). Lindleyana 11: 205-210

Toscano de Brito, A.L.V., 2001. Systematic rewiew of the Ornithocephalus group (Oncidiinae; Orchidaceae) with comments on Hofmeisterella. Lindleyana 16: 157-217.

Toscano de Brito, A.L.V. \& Kollman, L. 1997. Zygostates leptosepala: a new species in the subtribe Ornithocephalinae (Orchidaceae) from Brazil. Royal Botanic Gardens 52: 239-242.

Vale, A., Navarro, L., Rojas D. \& Álvarez, J.C. 2011. Breeding system and pollination by mimicry of the orchid Tolumnia guibertiana in Western Cuba. Plant Species Biology 26: 163-173. http://dx.doi. org/10.1111/j.1442-1984.2011.00322.x.

Vogel, S. 1974. Ölblumen und ölsammelnde Bienen. Tropische und Subtropische Pflanzenwelt 7: 285-547.

Vogel, S. 1988. Die Ölblumen - Symbiosen - Parallelismus und andere Aspekte ihrer Entwicklung in Raum und Zeit. Journal of Zoological Systematics and Evolutionary Research 26: 314-362.

Vogel, S. \& Cocucci, A.A., 1995. Pollination of Basistemon (Scrophulariaceae) by oil-collecting bees in Argentina. Flora 190 353-363.

Vogel, S. \& Machado, I.C. 1991. Pollination of four sympatric species of Angelonia (Scrophulariaceae) by oil-colecting bees in NE-Brazil. Plant Systematics and Evolution 178: 153-178.

Williams, L.O. 1939. Las Orchidaceae del noroeste argentino. Lilloa 4: $337-375$.

Associate Editor: Günter Gerlach Received: 29-XI-2013 Accepted: 2-IV-2014 